\title{
Parametric decay of beam-driven Langmuir wave and enhanced ion-acoustic fluctuations in the ionosphere: a weak turbulence approach
}

\author{
F. R. E. Forme \\ Centre d'étude des Environnements Terrestre et Planétaires, Velizy, France \\ Received: 5 October 1998 / Revised: 26 March 1999 / Accepted: 30 March 1999
}

\begin{abstract}
We present a model that describes the decay of beam generated Langmuir waves into ion-acoustic waves in the topside ionosphere. This calculation is done within the frame of the weak turbulence approximation. We study the spectral signature of such a process as seen by a VHF incoherent scatter radar. An incoherent scatter (IS) spectrum is characterized by two maxima at $\mathrm{k}_{\text {radar }}$ and $-\mathrm{k}_{\text {radar }}$, the right and left ion lines respectively. It is shown that, for reasonable beam parameters, the parametric decay of beam-generated Langmuir waves can enhance either the right, the left or both ion lines simultaneously. The shape of the spectrum can change drastically on time scale of about 0.1 to $1 \mathrm{~s}$. The role of the beam parameter as well as the ionospheric parameters is also investigated. For a given beam number density, the beam energy or the background density are important to trigger either the left or the right ion line. A large energy spread of the beam or low electron collision frequencies can explain the simultaneous observations of the left and the right ion line. The importance of the electron collision frequency can explain the altitude distribution of the coherent echoes observed by incoherent scatter radars.
\end{abstract}

Key words. Ionosphere (plasma waves and instabilities) · Space plasma physics (waves and instabilities; wavewave interactions)

\section{Introduction}

Powerful spectra have now been unambiguously observed with incoherent scatter radars both at UHF and VHF frequencies (Foster 1988; Collis et al., 1991; Rietveld et al., 1991). Such coherent spectra all exhibit one or two strong ion-acoustic peaks enhanced by one or two orders of magnitude. These echoes are observed along the magnetic field line, from the topside ionosphere all the way down to $140 \mathrm{~km}$. Rietveld et al. (1996) showed that such echoes statistically exhibit different features according to the altitude. For example, the upper shoulder (ion-acoustic waves going down) is enhanced more frequently than the lower shoulder. Above $300 \mathrm{~km}$ neither shoulder dominate the statistics. Also, low altitudes echoes are seldom observed. Collis et al. (1991) and Forme et al. (1995) showed that enhanced ion-acoustic fluctuations are strongly correlated with burst of soft electron $(<500 \mathrm{eV})$ precipitation. A complete description of these echoes is done by Fontaine and Forme (1995). A theoretical explanation for the generation of this enhanced acoustic echoes is still a matter of heated debate but two main explanations have emerged in the literature. Following Kindel and Kennel (1971), the first explanation involves relative drifts between the thermal species in the ionospheric plasma to excite the unstable waves. St-Maurice (1995) proposed that intense conductivity gradients in the presence of strong ambient electric fields could drive intense thermal currents along segments of the magnetic field. Although traces of intense field-aligned current exist (Stasiewicz et al., 1998), this model has several problems as presented by Kofman and St. Manice (1996).

A completely different approach has been advanced by Forme (1993). It was suggested that the enhanced ionacoustic fluctuations are due to the Langmuir wave decay $1 \rightarrow 1^{\prime}+\mathrm{s}$. In this process the "pump" Langmuir waves (l) are produced by a beam instability due to soft electrons beam of 10 to $500 \mathrm{eV}$. Those waves then produce backscattered Langmuir waves (l') and ionacoustic waves (s). They showed that for reasonable electron beam and ionospheric parameters this mechanism may occur in the upper ionosphere. This model predicts electric field amplitudes of about 1 to $200 \mathrm{mV} / \mathrm{m}$ for ion-acoustic and Langmuir waves respectively. Rough estimates of the beam parameter (velocity, velocity spread, density) were given using an analytical expression of the threshold electric field for the para- 
metric decay. However, this model in its present formulation, is not able to explain the whole observations like simultaneously propagating ion-acoustic waves in parallel and antiparallel (with respect to the magnetic field) direction. Also, such a simple model can not account for the different shapes of the spectra according to the altitude as shown by Rietveld et al. (1996).

The coupled dynamic of high-frequency Langmuir wave and ion-acoustic waves is described by the Zakharov equations (Zakharov, 1972). This set of equations describes the three-wave processes that result in the cascade of Langmuir energy to low wave numbers as well as the nucleation that result in the formation of coherent wave packets and their collapse in density wells. The weak turbulence approximation (WTA) is a statistical theory that describes the time evolution of the wave intensities via the wave kinetic equation (Sagdev and Galeev, 1969). It also includes the assumption that the waves have random phases (Kadomtsev, 1965). It is often assumed in WTA that the damping of the ionacoustic waves is so large that no significant enhancement of ion fluctuations occurs (Zakharov et al., 1989). However, a standard weak turbulence procedure that also involves the ion-acoustic dynamic have been presented by Nicholson (1983), Kadomtsev (1965), and Payne et al. (1989).

In this the wave kinetic equations in the frame of the WTA for both Langmuir and ion-acoustic waves are derived. This equations are used to model the decay of beam driven Langmuir waves into ion-acoustic waves for the high-latitude ionosphere. Finally, spectral signature on incoherent scatter radars VHF frequency is discussed as well as the role of the beam and the ionospheric parameters.

\section{The wave kinetic equation}

This study concentrates on the non-linear wave-wave interaction that involve ion-acoustic waves and Langmuir wave. The resonance conditions for this specific interaction are:

$\omega_{\mathrm{k}}^{l}=\omega_{\mathrm{k}^{\prime}}^{l}+\omega_{\mathrm{k}^{\prime \prime}}^{s}, \quad \mathrm{k}=\mathrm{k}^{\prime}+\mathrm{k}^{\prime \prime}$,

where the superscripts $s$ and $l$ stand for ion-sound and Langmuir waves, respectively. The two previous equations lead to (Tsytovich, 1970):

$\mathrm{k}^{\prime \prime}=2 \mathrm{k}^{\prime}-\frac{2 \omega_{p, e} C_{s}}{3 \mathrm{v}_{e}^{2}}$,

where $C_{s}$ is the ion sound velocity and $\mathrm{v}_{e}$ the thermal velocity. The wave kinetic equation that describes the time evolution of the ensemble-averaged spectral intensity of the $\alpha$ th mode can be written as (Sagdeev and Galeev, 1969):

$\frac{\partial}{\partial t} N_{\mathrm{k}}^{\alpha}=2 \gamma_{\mathrm{k}}^{\alpha} N_{\mathrm{k}}^{\alpha}+\int_{-\infty}^{+\infty} M_{\mathrm{k}, \mathrm{k}^{\prime}}^{\alpha} \mathrm{dk}^{\prime}$

where $M_{\mathrm{k}, \mathrm{k}^{\prime}}^{\alpha}$ is the function that account for the coupling between the interacting triplet $(\alpha, \mathrm{k}),\left(\alpha, \mathrm{k}^{\prime}\right),\left(\beta, \mathrm{k}^{\prime \prime}\right)$ and $\gamma$ is the damping/growth of the corresponding mode. The function $M_{\mathrm{k}, \mathrm{k}^{\prime}}^{\alpha}$ is calculated within the framework of weak turbulence theory (Sagdeev and Galeev, 1969). Since we want to include the ion dynamics in that particular model we use the application of the weak turbulence theory adopted by Nicholson (1983), as described briefly.

The version of the one-dimensional Zakharov system of equations that are used in this work reads:

$$
\begin{aligned}
& \left(i \frac{\partial}{\partial t}+\frac{\partial^{2}}{\partial x^{2}}\right) E=n E \\
& \left(\frac{\partial^{2}}{\partial t^{2}}-\frac{\partial^{2}}{\partial x^{2}}\right) n=\frac{\partial^{2}}{\partial x^{2}}|E|^{2} .
\end{aligned}
$$

Equation (2) describes the slow evolution of the Langmuir envelope $E(x, t)$. The left-hand side describes the linear evolution while the right-hand side accounts for the nonlinear effects due to density perturbations $n(x, t)$. Similarly, the left-hand side of Eq. (3) is the linear behavior of ion-acoustic waves and its right-hand side represents the nonlinear effect of the ponderomotive force which drives electrons and ions out of regions of high-field. Kinetic effects can be included in Eqs. (2) and (3) through a collisional term. Dimensionless variables are used that are related to their dimensional counterparts (denoted by a tilde) by $t=\tilde{t} / \tau, x=\tilde{x} / \chi, E=\tilde{E} / \epsilon$, and $n=\tilde{n} / v$, where

$$
\begin{aligned}
& \tau=\frac{3 m_{i}}{2 \eta m_{e}} \tilde{\omega}_{p, e}^{-1} \quad \chi=\left(\frac{9 m_{i}}{4 \eta m_{e}}\right)^{1 / 2} \tilde{\lambda}_{e}, \\
& \epsilon=\left(\frac{16 m_{e} \eta}{3 m_{i} \epsilon_{0}}\right)^{1 / 2}\left(n_{0} \mathrm{k}_{b} T_{e}\right)^{1 / 2} \quad v=\frac{4 \eta m_{e}}{3 m_{i}} .
\end{aligned}
$$

Here $\eta=\left(\gamma_{e} T_{e}+\gamma_{i} T_{i}\right) / T_{e}, \gamma_{e}$ and $\gamma_{i}$ are the ratio of specific heat. Using this normalization, the dimensional Langmuir wave and ion-acoustic wave dispersion relations become $\omega_{\mathrm{k}}^{l}=\mathrm{k}^{2}$ and $\omega_{\mathrm{k}}^{s}=|\mathrm{k}|$, respectively. The spatial Fourier transform of Eqs. (2) and (3) gives:

$$
\begin{aligned}
& \left(i \frac{\partial}{\partial t}-\mathrm{k}^{2}\right) E_{\mathrm{k}}=\int_{-\infty}^{+\infty} \mathrm{dk}^{\prime} n_{\mathrm{k}^{\prime}} E_{\mathrm{k}-\mathrm{k}^{\prime}} \\
& \left(\frac{\partial^{2}}{\partial t^{2}}+\mathrm{k}^{2}\right) n_{\mathrm{k}}=-\mathrm{k}^{2} \int_{-\infty}^{+\infty} \mathrm{dk}^{\prime} E_{\mathrm{k}^{\prime}} E_{\mathrm{k}^{\prime}-\mathrm{k}}^{*} .
\end{aligned}
$$

Here $E_{\mathrm{k}}$ and $n_{\mathrm{k}}$ are time-dependent variables. The linear limit of Eqs. (4) and (5) can be found, ignoring the nonlinear right side of the equations. The idea of the weak turbulence approximation is to assume that both Langmuir waves and ion sound waves obey their linear solution. However a slow time variation of their amplitude is allowed because of the nonlinear term on the right side of Eqs. (4) and (5). Therefore, we want to solve Eqs. (4) and (5) with a solutions of the form:

$$
\begin{aligned}
& E_{\mathrm{k}}=\bar{E}_{\mathrm{k}} \exp \left(-i \omega_{\mathrm{k}}^{l} t\right) \\
& n_{\mathrm{k}}=A_{\mathrm{k}} \exp \left(-i \omega_{\mathrm{k}<0}^{s} t\right)+B_{\mathrm{k}} \exp \left(-i \omega_{\mathrm{k}>0}^{s} t\right)
\end{aligned}
$$


where $\bar{E}_{\mathrm{k}}, A_{\mathrm{k}}$ and $B_{\mathrm{k}}$ are slowly varying. In Eq. (7) the first term on the right side represents up-going ionacoustic waves while the second term represents downgoing ion-acoustic waves. In the following the quantities of interest are:

$N_{\mathrm{k}}^{l} \delta\left(\mathrm{k}-\mathrm{k}^{\prime}\right)=\left|\bar{E}_{\mathrm{k}}\right|^{2} \delta\left(\mathrm{k}-\mathrm{k}^{\prime}\right)=\left\langle\bar{E}_{\mathrm{k}} \bar{E}_{\mathrm{k}^{\prime}}^{*}\right\rangle$

$N_{\mathrm{k}}^{s} \delta\left(\mathrm{k}-\mathrm{k}^{\prime}\right)=\left|n_{\mathrm{k}}\right|^{2} \delta\left(\mathrm{k}-\mathrm{k}^{\prime}\right)=\left\langle C_{\mathrm{k}} C_{\mathrm{k}^{\prime}}^{*}\right\rangle$

where $C_{\mathrm{k}}$ is defined as:

$C_{\mathrm{k}}=\left\{\begin{array}{ll}A_{\mathrm{k}}, & \mathrm{k}<0 \\ B_{\mathrm{k}}, & \mathrm{k}>0\end{array}\right.$.

In doing so, we assume that the wave phases are distributed randomly when considering an ensemble of experiments (Sagdeev and Galeev, 1969). Using the standard weak turbulence procedure (Nicholson, 1983; Kadomtsev, 1965; Payne et al., 1989) we construct the function $M_{\mathrm{k}, \mathrm{k}^{\prime}}^{\alpha}$. The result is:

$$
\begin{aligned}
M_{\mathrm{k}, \mathrm{k}^{\prime}}^{l}= & {\left[\left(N_{\mathrm{k}-\mathrm{k}^{\prime}}^{l}-N_{\mathrm{k}}^{l}\right) N_{\mathrm{k}^{\prime}}^{s}-\frac{\mathrm{k}^{\prime 2}}{2 \omega_{\mathrm{k}^{\prime}}^{s}} N_{\mathrm{k}}^{l} N_{\mathrm{k}-\mathrm{k}^{\prime}}^{l}\right] } \\
& \times \delta\left(\omega_{\mathrm{k}}^{l}-\omega_{\mathrm{k}^{\prime}}^{s}-\omega_{\mathrm{k}-\mathrm{k}^{\prime}}^{l}\right) \\
& +\left[\left(N_{\mathrm{k}+\mathrm{k}^{\prime}}^{l}-N_{\mathrm{k}}^{l}\right) N_{\mathrm{k}^{\prime}}^{s}+\frac{\mathrm{k}^{\prime 2}}{2 \omega_{\mathrm{k}^{\prime}}^{s}} N_{\mathrm{k}}^{l} N_{\mathrm{k}+\mathrm{k}^{\prime}}^{l}\right] \\
& \times \delta\left(\omega_{\mathrm{k}}^{l}+\omega_{\mathrm{k}^{\prime}}^{s}-\omega_{\mathrm{k}+\mathrm{k}^{\prime}}^{l}\right)
\end{aligned}
$$

and

$$
\begin{aligned}
M_{\mathrm{k}, \mathrm{k}^{\prime}}^{s}= & \frac{\pi \mathrm{k}^{2}}{\omega_{\mathrm{k}}^{s}}\left[\left(N_{\mathrm{k}}^{s}+\frac{\mathrm{k}^{2}}{2 \omega_{\mathrm{k}}^{s}} N_{\mathrm{k}^{\prime}-\mathrm{k}}^{l}\right) N_{\mathrm{k}^{\prime}}^{l}+\left(N_{\mathrm{k}^{\prime}}^{l}-N_{\mathrm{k}^{\prime}-\mathrm{k}}^{l}\right) N_{\mathrm{k}}^{s}\right] \\
& \times \delta\left(\omega_{\mathrm{k}^{\prime}}^{l}-\omega_{\mathrm{k}^{\prime}-\mathrm{k}}^{l}-\omega_{\mathrm{k}}^{s}\right)
\end{aligned}
$$

In deriving Eqs. (8) and (9) from Eqs. (2) and (3) we make a transition from a dynamical to a statistical description of the turbulence (Nicholson, 1983; Kadomtsev, 1965). Henceforth, information on the Langmuir and acoustic wave phases is lost since the weak turbulence approximation only describe the wave intensities. It has been found that the random phase approximation (RPA) is justified when the backscattered waves are not effectively phase-locked to the driver wave (Robinson et al., 1992).

\section{The model}

We want to solve the system of coupled differential equations:

$$
\begin{aligned}
& \frac{\partial}{\partial t} N_{\mathrm{k}}^{l}=2 \gamma_{\mathrm{k}}^{l} N_{\mathrm{k}}^{l}+\int_{-\infty}^{+\infty} M_{\mathrm{k}, \mathrm{k}^{\prime}}^{l} \mathrm{dk}^{\prime} \\
& \frac{\partial}{\partial t} N_{\mathrm{k}}^{s}=2 \gamma_{\mathrm{k}}^{s} N_{\mathrm{k}}^{s}+\int_{-\infty}^{+\infty} M_{\mathrm{k}, \mathrm{k}^{\prime}}^{s} \mathrm{dk}^{\prime}
\end{aligned}
$$

which are coupled through the functions $M_{\mathrm{k} . \mathrm{k}^{\prime}}^{l}$ and $M_{\mathrm{k}, \mathrm{k}^{\prime}}^{s}$. Integrals in Eqs. (10) and (11) are straightforward if the dispersion relations of Langmuir and ion-acoustic waves are used to express the delta functions. It is often assumed that the damping of the ion-acoustic waves is so large that no significant enhancement of ion fluctuations occurs. Therefore Eq. (11) is usually forgotten (Zakharov et al., 1989). The damping terms are used as phenomenological models of the wave-particles interactions. The Langmuir damping term includes: a negative damping to account for the beam instability $\gamma_{b}$, and the sum of collisions and linear Landau damping of Langmuir wave $\gamma_{L}$. A comprehensive study of the beam plasma instability has been given by Cairns (1987). The growth rate is given by Ichimaru (1973):

$\gamma_{b}=\left.\frac{\chi}{\tau} \frac{\pi}{2 n} \frac{\omega_{p, e}^{3}}{\mathrm{k}^{2}} \frac{\partial}{\partial \mathrm{v}} F_{b}\right|_{\omega_{p, e} / \mathrm{k}}$,

where $F_{b}$, the beam distribution function is given by:

$F_{b}=\frac{n_{b}}{\sqrt{\pi} \Delta \mathrm{v}_{b}} \exp \left[-\left(\frac{\mathrm{v}_{b}-\mathrm{v}}{\Delta \mathrm{v}_{b}}\right)^{2}\right]$.

Hence, the growth rate can easily be expressed in terms of the beam parameter:

$$
\begin{aligned}
\gamma_{b}= & \frac{\chi}{\tau} \sqrt{\pi}\left(\frac{\omega_{p, e}}{\mathrm{k} \Delta \mathrm{v}_{b}}\right)^{2} \omega_{p, e} \frac{n_{b}}{n} \frac{\mathrm{v}_{b}-\omega_{p, e} / \mathrm{k}}{\Delta \mathrm{v}_{b}} \\
& \times \exp \left[-\left(\frac{\mathrm{v}_{b}-\omega_{p, e} / \mathrm{k}}{\Delta \mathrm{v}_{b}}\right)^{2}\right] .
\end{aligned}
$$

In the following, $\mathrm{k}_{b}$ is defined as $\gamma\left(\mathrm{k}_{b}\right)=0$ which corresponds to $\mathrm{k}_{b}=\omega_{p, e} / \mathrm{v}_{b}$. Such an expression of the beam growth rate has been preferred to the usual Gaussian expression of the type:

$\Gamma_{b} \exp \left[-\left(\frac{\mathrm{v}_{b}-\omega_{p, e} / \mathrm{k}}{\Delta \mathrm{v}_{b}}\right)^{2}\right]$

where $\Gamma_{b}$ is a constant, because Eq. (12) gives a strong damping at $\mathrm{k}<\mathrm{k}_{b}$. This damping is due to the negative slope of the beam distribution function at $\mathrm{v}>\mathrm{v}_{b}$. The expected effect of such a growth rate is to inhibit or reduce the cascade of Langmuir energy to low wave numbers. It is also well known that the high frequency waves enhanced by such an instability can react back on the distribution modifying it in such a way as to reduce the growth of the unstable waves. This process is known as quasi-linear diffusion of particles in velocity space. In order to take into account this effect in our model the growth rate is truncated at a level $\gamma_{\max }$ (Newmann et al., 1994). It is by means of this truncation that we model in a non-self-consistent way the effects of wave-particle quasi-linear interaction. The dimensionless collisional and Landau damping term is given by:

$\gamma_{L}=-\frac{v_{e}}{2}-\sqrt{\frac{\pi}{8}}\left(\frac{m_{i}}{\eta m_{e}}\right)^{5 / 2} \frac{9}{4} \frac{1}{|\mathrm{k}| \mathrm{k}^{2}} \exp \left(-\frac{9}{8} \frac{m_{i}}{\eta m_{e}} \frac{1}{\mathrm{k}^{2}}-\frac{3}{2}\right)$,

where $v_{e}$ is the electron collision frequency. The electron collision frequency can vary by orders of magnitude in the ionosphere depending mainly on the electron temperature and the electron density. Hence, $v_{e}$ is an 
important parameter since it determine whether the Langmuir instability is triggered or not. To summarize, the damping of the Langmuir mode is expressed as:

$\gamma_{\mathrm{k}}^{l}=\gamma_{L}+\min \left(\gamma_{\max }, \gamma_{b}\right)$

The ion-acoustic damping term, $\gamma_{\mathrm{k}}^{s}$ accounts for the linear Landau damping. It has been numerically estimated by solving the ion-acoustic dispersion relation using WHAMP (Rönmark, 1982). We solve Eqs. (10) and (11) with a standard Runge-Kutta method using 2048 modes. We applied initial conditions which correspond to a cold start. We start with a background noise level for the Langmuir electric field and the ion density fluctuations (i.e. $\bar{E}_{\mathrm{k}}=10^{-2}$ ). Similar values of the background noise level have been use by Hansen et al. (1992) and Newmann et al. (1994).

The input parameters are the beam energy $E_{b}$, the beam number density $n_{b}$, the energy dispersion $\Delta E_{b} / E_{b}$, the background density $n_{0}$ and the collision frequency $v_{e}$. All the calculations have been done for $\mathrm{O}^{+}$ions, a temperature ratio $T_{e} / T_{i}=2$ and $T_{e}=2000 \mathrm{~K}$ and $\gamma_{\max }=$ $400 \tau\left(\tilde{\gamma}_{\max }=2.24 \times 10^{-5} \tilde{\omega}_{p, e}\right)$. This value of the saturated growth rate is quite arbitrary but changing this value does not modify the conclusions of this study.

\section{Results}

The first calculation has been made for $\tilde{n}_{0}=10^{11} \mathrm{~m}^{-3}$, $\tilde{n}_{b}=2 \times 10^{4} \mathrm{~m}^{-3}, \tilde{E}_{b}=45 \mathrm{eV}, \Delta \tilde{E}_{b} / \tilde{E}_{b}=1 / 4$ and $\tilde{v}_{e}=$ $200 \mathrm{~s}^{-1}$. The beam energy selects the wave number $\left(\mathrm{k}_{\max }\right)$ of the driven Langmuir mode while the wave vector of the unstable ion-acoustic fluctuations is determined by $\mathrm{k}_{\tilde{\mathrm{N}}}=2 \mathrm{k}_{\max }-1$. Such beam parameters, gives $\mathrm{k}_{b} \approx 7.1\left(\tilde{\mathrm{k}}_{b} \tilde{\lambda}_{e} \approx 0.042\right)$ and a maximum growth rate at $\mathrm{k}_{\max } \approx 7.7\left(\tilde{\mathrm{k}}_{\max } \tilde{\lambda}_{e} \approx 0.048\right)$. They have been chosen for the wave vector of the enhanced ion-acoustic fluctuations to roughly match the backscattered wave vector of the EISCAT VHF radar $\mathrm{k}_{V H F}=9.4 \chi \approx 14.9$ $\left(\tilde{\mathrm{k}}_{V H F} \tilde{\lambda}_{e} \approx 0.0928\right)$.

The time history of the spectral intensity of the Langmuir waves is presented in Fig. 1. The beam-driven modes, excited on a bandwidth of about $\Delta \mathrm{k} \approx 0.8$ $\left(\Delta \tilde{\mathrm{k}} \tilde{\lambda}_{e} \approx 0.005\right)$, are seen at $\mathrm{k} \approx 8\left(\tilde{\mathrm{k}} \tilde{\lambda}_{e} \approx 0.05\right)$. When the energy in the driven modes exceeds the threshold, at $t=87\left(\tilde{t} \tilde{\omega}_{p, e}=1.53 \times 10^{6}\right)$, the three-wave decay of the form $1 \rightarrow 1^{\prime}+s$ operates. This process transfers the energy out of the beam-driven modes to the backscatter modes. This results in a second region of enhanced intensities at $\mathrm{k} \approx-6.8\left(\tilde{\mathrm{k}} \tilde{\lambda}_{e} \approx-0.042\right)$. For the parameters that we have used in this calculation, the cascade is inhibited to the first backscatter. A quasiperiodic behavior is clearly seen on both the beamdriven and the backscattered modes with the period decreasing from $T=67\left(\tilde{T} \tilde{\omega}_{p, e}=1.17 \times 10^{6}\right)$ to $T=57$ $\left(\tilde{T} \tilde{\omega}_{p, e}=10^{6}\right)$.

Figure 1 also shows the time history of the density perturbation spectrum for the same parameters in Figure 1 (upper panel). No ion-acoustic waves are seen before the three-wave decay starts at $t=87$ $\left(\tilde{t} \tilde{\omega}_{p, e}=1.53 \times 10^{6}\right)$. Thereafter, one region of enhanced density fluctuations is seen at $\mathrm{k} \approx \pm 14.5\left(\tilde{\mathrm{k}} \tilde{\lambda}_{e} \approx \pm 0.09\right)$ which corresponds to about $\pm 2 \mathrm{k}_{\max }-1$. If the electron beam is thought of as a downward propagating beam (i.e. $\mathrm{k}>0$ ), the region of maximum intensities represents down-going ion-acoustic waves. The density perturbation spectrum presents a quasi-periodic behavior with a maximum intensity of $10^{-2}$. The bandwidth of the excited ion-acoustic spectrum is about twice the bandwidth of the beam- driven modes.

The time evolution of the spatially averaged dimensionless wave energy density:

$$
\langle W\rangle=\frac{\epsilon_{0}\left\langle|E|^{2}\right\rangle}{n_{0} \mathrm{k}_{b} T_{e}}
$$

is shown on Fig. 2. At $t=0\langle W\rangle=4 \times 10^{-6}$. The quasiperiodic fluctuations are clearly seen around a mean value of about $\langle W\rangle=6 \times 10^{-5}$. The amplitude of the fluctuations are decreasing with time. One should be aware that a radar experiment observes height-integrated frequency spectra due to density fluctuations at the radar backscatter wave number, $\mathrm{k}_{\text {radar }}$. An incoherent scatter (IS) spectrum is characterized by two maxima at $\mathrm{k}_{\text {radar }}$ and $-\mathrm{k}_{\text {radar }}$, the right and left ion lines respectively. Therefore a qualitative estimate of the relative importance of the two ion lines may be found by plotting the density spectrum at the radar wave vector. Since the beam parameters have been chosen to enhanced ion-acoustic fluctuations at the VHF wave number, $\mathrm{k}_{V H F}$, we will present results concerning the VHF spectrum (i.e. $\left|n_{\mathrm{k}_{V H F}}\right|^{2}$ ). Figure 2 shows the temporal evolution of the density fluctuations at the VHF wave vector components. The solid line represents $\left|n_{\mathrm{k}}\right|^{2}$ at $\mathrm{k}=\mathrm{k}_{V H F}$ and the dashed line represents $\left|n_{\mathrm{k}}\right|^{2}$ at $\mathrm{k}=-\mathrm{k}_{V H F}$, corresponding respectively to down-going and up-going ion-acoustic waves. Only one line is enhanced since we have already seen that the cascade is inhibited after the first backscattered Langmuir wave. The enhancements have a very short duration. This line reaches a maximum value of 0.01 at the beginning of the calculation while its initial value was $10^{-4}$. The second ion line stays at its initial level.

In the integration time an IS radar experiment sums the fluctuations seen on the left and the right ion line, so we define the relative amplitude of the two ion lines as:

$$
\rho(t)=\frac{\int_{0}^{t}\left|n_{\mathrm{k}_{V H F}}\right|^{2} \mathrm{~d} t^{\prime}}{\int_{0}^{t}\left|n_{-\mathrm{k}_{H F}}\right|^{2} \mathrm{~d} t^{\prime}} .
$$

In this definition, $t^{\prime}=0$ means the beam turn on time. Since this time does not correspond to the beginning of the integration time for the IS spectrum, one should be careful in interpreting this parameter. Therefore, the following values correspond to the maximum values of the asymmetry between the two ion lines. This should occur if the phenomenum described here operates during the whole time integration. A beam duration shorter than the integration time of the IS radar will result in a reduced asymmetry of the ion lines. The result for the same parameters as Fig. 1 is shown in Fig. 2. Since only the right ion line is enhanced (i.e. $\left|n_{\mathrm{k}}\right|^{2}$ at 

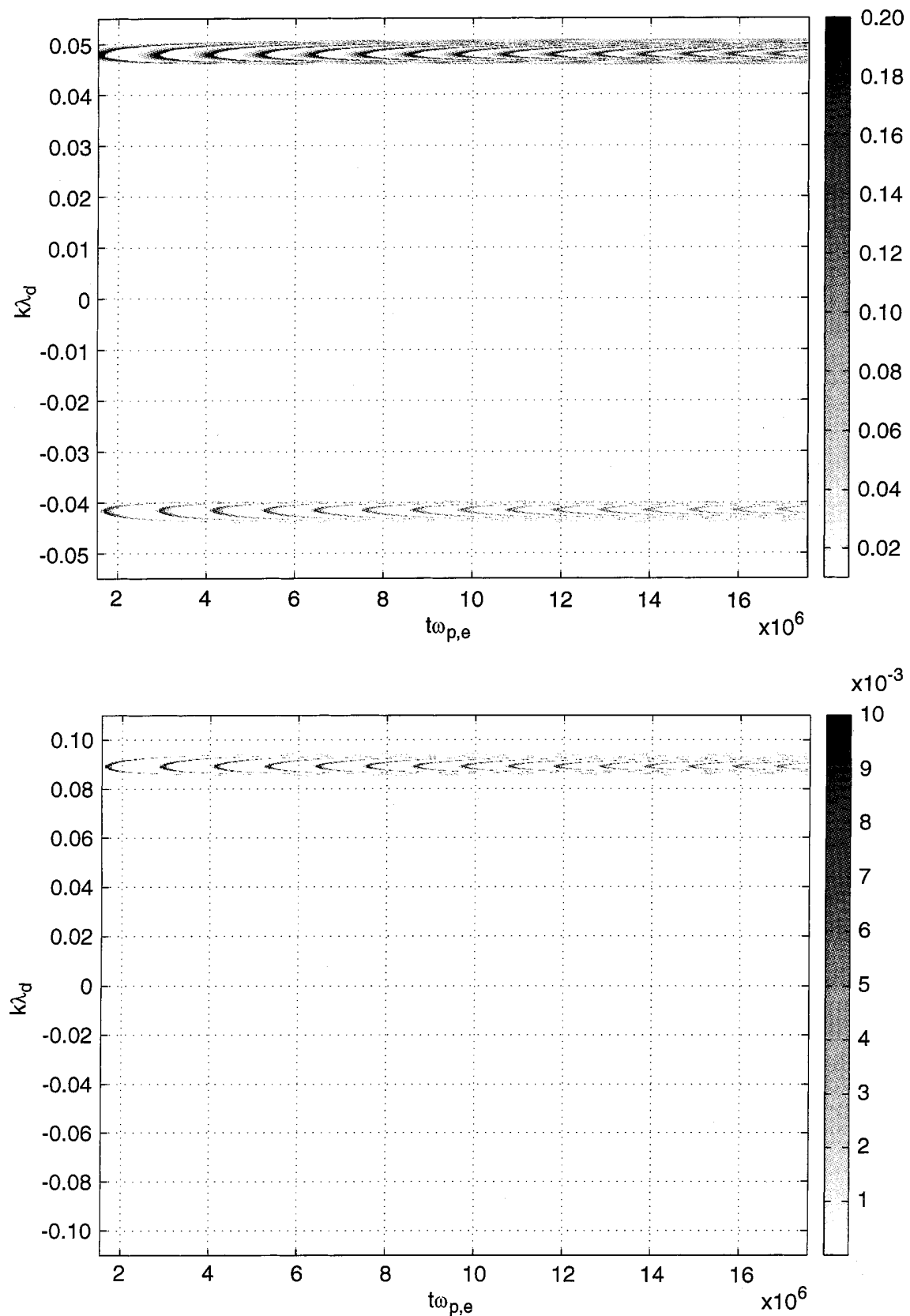

Fig. 1. Time history of the spectral intensity of the Langmuir waves $\left|E_{\mathrm{k}}\right|^{2}$ (upper panel) and the density perturbation spectrum for $\tilde{n}=10^{11} \mathrm{~m}^{-3}, \tilde{n}_{b}=2 \times 10^{4} \mathrm{~m}^{-3}, \widetilde{E}_{b}=45 \mathrm{eV}$ $\Delta \tilde{E}_{b} / \tilde{E}_{b}=1 / 4$ and $\tilde{v}_{e}=200 \mathrm{~s}^{-1}$

$\left.\mathrm{k}=\mathrm{k}_{V H F}\right)$ the ratio $\rho>0$. Although moderate, the maximum asymmetry between the two ion lines is seen at the beginning of the calculation. The shape of the spectrum can change drastically on very short time scale. On longer time scale, e.g. $t>500\left(\tilde{t} \tilde{\omega}_{p, e}>8.7 \times 10^{6}\right)$, the right ion line is preferentially enhanced with a relative amplitude that stabilizes about $\rho \approx 10$.

Another calculation has been completed by using a beam energy $\tilde{E}_{b}=30 \mathrm{eV}$ and a beam number density of $\tilde{n}_{b}=6 \times 10^{4} \mathrm{~m}^{-3}$, all other input parameters remaining unchanged. Increasing the beam number density will increase the beam growth rate and therefore allows several backscatters, although this is partly balanced by the decrease of the beam energy. Hence we will have ionacoustic waves propagating in both direction parallel and anti-parallel to the electron beam. Also the band- width (not shown here) of the excited Langmuir waves, and consequently the backscatters, is larger than in the previous case. For this beam density there are no backscatters beyond the second in which there is very little energy. While the beam excited waves are centered at $\mathrm{k} \approx 9.5\left(\tilde{\mathrm{k}} \tilde{\lambda}_{e} \approx 0.059\right)$ the first and second backscatters are at about $\mathrm{k} \approx-8.5\left(\tilde{\mathrm{k}} \tilde{\lambda}_{e} \approx-0.053\right)$ and $\mathrm{k} \approx 7.5$ $\left(\tilde{\mathrm{k}} \tilde{\lambda}_{e} \approx 0.047\right)$. This gives rise to two regions of enhanced density fluctuations centered about $\mathrm{k} \approx 18\left(\tilde{\mathrm{k}} \tilde{\lambda}_{e} \approx 0.112\right)$ and $\mathrm{k} \approx-16\left(\tilde{\mathrm{k}} \tilde{\lambda}_{e} \approx 0.1\right)$. Figure 3 shows the total electrostatic Langmuir energy, the density fluctuations at the radar backscatter wave number and the relative amplitude of the two ion lines for this calculation. The electrostatic Langmuir energy oscillates with a shorter period than in the first calculation of about $T=20$ $\left(\tilde{T} \tilde{\omega}_{p, e}=3.5 \times 10^{5}\right)$ around a mean value of about 240 , 

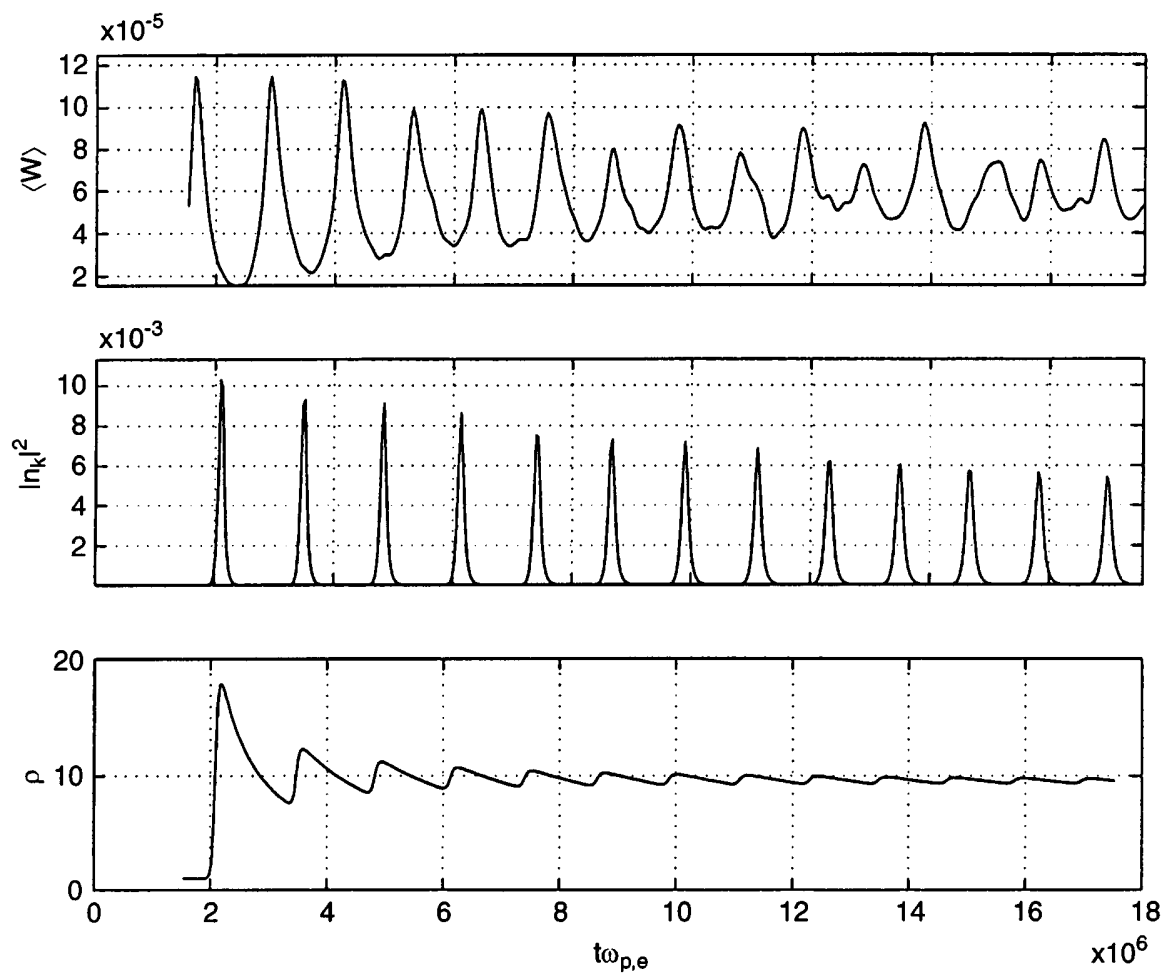

Fig. 2. The time evolution of: (upper panel) the spatially averaged dimensionless wave energy density $\langle W\rangle$, middle panel, the density fluctuations at the VHF wave vector components (lower panel) and the relative amplitude of the two ion lines. The input parameters are the same as Fig. 1 one order of magnitude larger than in the previous case. Only the left ion line is enhanced in this case (Fig. 3, middle panel). Although, none of the region of enhanced density fluctuations is centered on $\pm \mathrm{k}_{V H F}$, the second part of the ion spectrum, centered about $\mathrm{k}=-16$ $\left(\tilde{\mathrm{k}} \tilde{\lambda}_{e} \approx 0.1\right)$, has a bandwidth that gives rise to an enhanced ion line at $\mathrm{k}=-\mathrm{k}_{V H F}$. As expected, this gives a relative amplitude of about $\rho \approx 10^{-2}$ for $T<200$ $\left(\tilde{t} \tilde{\omega}_{p, e}<3.51 \times 10^{6}\right)$ and $\rho \approx 10^{-1}$ at a later time. In this case the enhancement of the left ion line is the about the same as the enhancement of the right ion line in the previous calculation.

The last calculation was made for $\tilde{n}_{b}=10^{5} \mathrm{~m}^{-3}$, $\Delta \tilde{E}_{b} / \tilde{E}_{b}=1 / 3$ the other parameters remaining unchanged
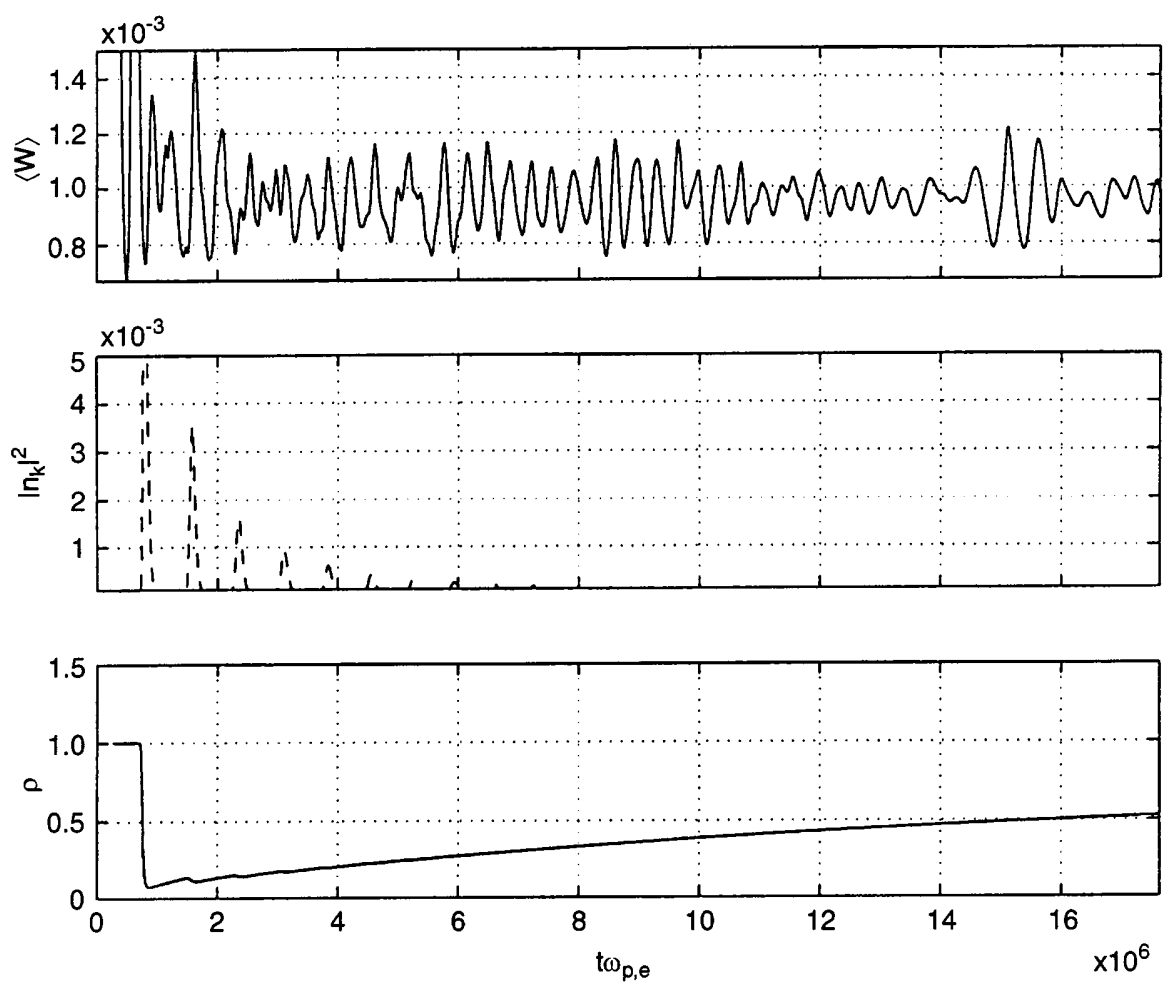

Fig. 3. Same as Fig. 2 for a beam energy $E_{b}=30 \mathrm{eV}$ and a beam number density of $\tilde{n}_{b}=6 \times 10^{4} \mathrm{~m}^{-3}$, all other input parameters remaining unchanged 

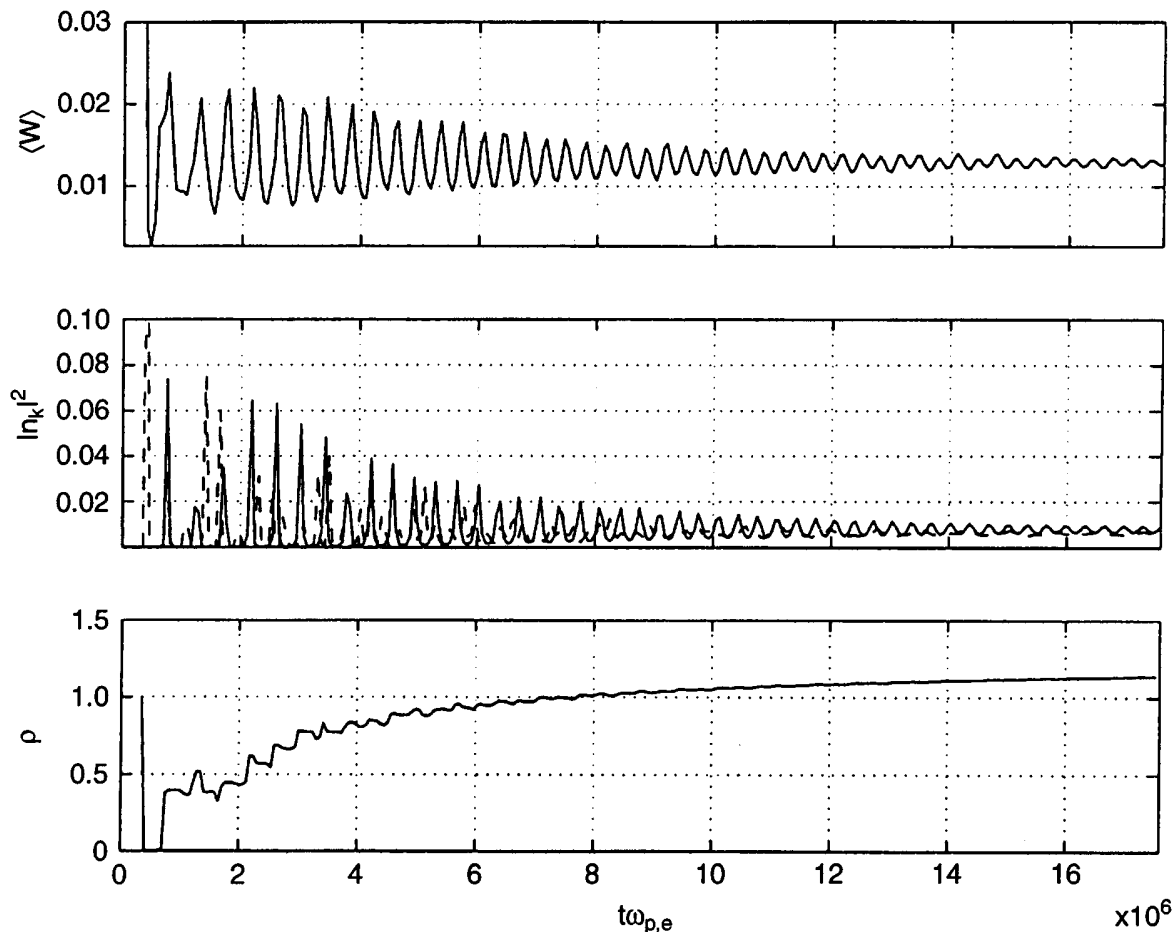

Fig. 4. Same as Fig. 2 for $\tilde{n}_{b}=10^{5} \mathrm{~m}^{-3}$, $\Delta \tilde{E}_{b} / \tilde{E}_{b}=1 / 3$ compared to the first example. The energy spread has been increased to give a larger bandwidth than in the two previous cases. Since, this diminishes the growth rate of the beam plasma instability we increased the beam number density in order to have two backscatters and the same growth rate as in the previous run. The total electrostatic energy (Fig. 4) is enhanced by 2 orders of magnitude compared to the first calculation. The quasi period is $T=21\left(\tilde{T} \tilde{\omega}_{p, e}=3.5 \times 10^{5}\right)$ and remains almost constant during the whole calculation. Since we enlarged the bandwidth of the excited waves this allows the parts of the ion spectrum corresponding to the first and second backscatter to match $\mathrm{k}_{V H F}$ and $-\mathrm{k}_{V H F}$

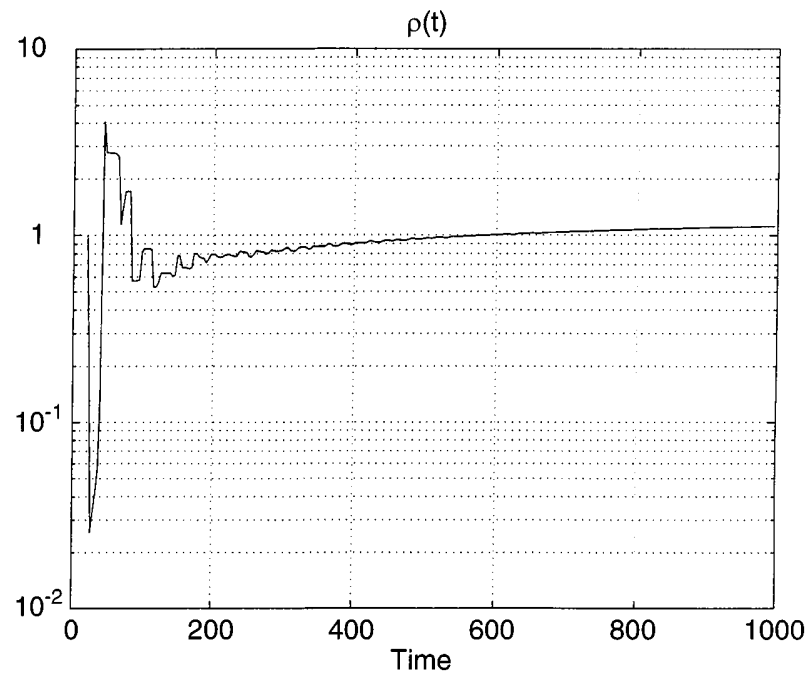

Fig. 5. Relative amplitude of the two ion lines for the same parameters as Fig. 4 except that the beam energy is $E_{b}=47 \mathrm{eV}$ simultaneously. It gives rise to enhancements of the two ion lines, shown on Fig. 4. The left ion line dominates at the beginning of the calculation. At $t>400 \tilde{t} \tilde{\omega}_{p, e}=7 \times 10^{6}$ the right ion line weakly dominates (i.e. $\rho>1$ ). The relative amplitude of the two ion line is very sensitive to the beam parameters and especially to the beam energy. Figure 5 shows the parameter $\rho$ for the same beam as Fig. 4 except that the energy is $\tilde{E}_{b}=47 \mathrm{eV}$ instead of $\tilde{E}_{b}=45 \mathrm{eV}$. One may see that the ion spectra may be either left-enhanced or rightenhanced depending on the time when the three wave processes stop.

\section{Discussion}

The results presented are valid within the frame of the weak turbulence. It is therefore of importance to check the validity of the parameters we have used. The transition between weak turbulence and coexisting weak and strong turbulence occurs for Robinson et al. (1992):

$\frac{\Gamma}{v_{e}} \simeq \mathrm{k}_{\max }-1$

where $\Gamma=\max \left(\gamma_{\mathrm{k}}^{l}\right)$. This gives a transition at about $\Gamma / v_{e} \approx 7$ which was never exceeded in our calculations. Also a numerical test of the weak turbulence theory has been done by Payne et al. (1989). They compared numerical solutions of the statistical weak turbulence theory with numerical solutions of the Zakharov model of Langmuir turbulence. They derived the two following validity conditions for the weak turbulence theory:

$\sum_{\mathrm{k} \in \mathrm{k}_{n}}\left|E_{\mathrm{k}}\right|^{2} \ll\left(\Delta \mathrm{k}_{n}^{l}\right)^{2}$ 
$\tau_{c}^{2} \sum_{\mathrm{k}}\left|n_{\mathrm{k}}\right|^{2} \Delta \mathrm{k}^{s} \ll 1$

$\Delta \mathrm{k}_{n}^{l}$ is the width of the cascade line number $n, \Delta \mathrm{k}^{s}$ is the width of the low frequency spectrum and $\tau_{c}$ is the correlation time. $\tau_{c}$ is given by:

$\tau_{c} \sim \frac{1}{\left|1-2\left(\mathrm{k}+\mathrm{k}_{0}\right)\right| \Delta \mathrm{k}^{s}}$,

where $\mathrm{k}_{0}$ is the center of the ion acoustic spectrum of width $\Delta \mathrm{k}^{s}$. The validity criteria can be further approximated if it is assumed that the cascade line and the ion acoustic spectrum have a triangular shape. This allows us to formulate Eqs. (14) and (15) as:

$\left|E_{\mathrm{k}}\right|^{2} \ll \Delta \mathrm{k}_{n}^{l}$

$\tau_{c}^{2}\left|n_{\mathrm{k}}\right|^{2}\left(\Delta \mathrm{k}^{s}\right)^{2} \ll 1$

For the calculations presented, $\Delta \mathrm{k}_{n}^{l} \approx 1, \Delta \mathrm{k}^{s} \approx 1, \mathrm{k} \approx 10$ and $\mathrm{k}_{0} \approx 20$ which gives $\tau_{c} \approx(40)^{-1}$ and the validity criteria become: $\left|E_{\mathrm{k}}\right|^{2} \ll 1$ and $\left|n_{\mathrm{k}}\right|^{2} \ll 60$. Consequently, all cases presented in this study are believed to come under a weak turbulence approach.

Beam parameters pertaining to Langmuir waves in the auroral regions have been presented by e.g. $\mathrm{Mu}-$ schietti et al. (1994), Newman et al. (1994) and Forme (1993). The beam parameters we have used are well within the limits given by these authors. The plasma parameters are typical parameters of the ionospheric F-region where coherent echoes are mainly detected. Typical temperature ratios are $T_{e} / T_{i}=2-4$. We have chosen the lower limit to give a hard damping of the ionacoustic wave and therefore to lead to maximum values for the beam parameters.

The quasi-periodic behavior in beam-driven Langmuir turbulence has been well explained by Robinson et al. (1992). They showed that a simple Lotka-Volterra model gives an excellent description of the periodic evolution. However they used a strong driver for the Langmuir turbulence and consequently they had large amplitude oscillations while we obviously have small amplitudes. If the ion-acoustic waves are neglected, making the approximation $N^{s} \ll N^{l}$, we can write Eq. (10) for the driven and backscatter Langmuir waves,

$$
\frac{\partial}{\partial t} N^{l}=2 \Gamma N^{l}-\alpha N^{l} N^{l^{\prime}}
$$

$\frac{\partial}{\partial t} N^{l^{\prime}}=-v_{e} N^{l^{\prime}}+\alpha N^{l} N^{l^{\prime}}$

where $\alpha$ is a constant. Equations (19) and (20) have steady state solutions: $N^{l^{\prime}}=\Gamma / \alpha$ and $N^{l}=v_{e} / \alpha$, and the small amplitude oscillations about this solution have the period $T_{0}=\pi / \sqrt{\Gamma v_{e} / 2}$. For the case shown in Fig. 1 where $T=67\left(\tilde{t} \tilde{\omega}_{p, e}=1.17 \times 10^{6}\right), \Gamma=4.5 \times 10^{-2}$ $\left(\tilde{\Gamma} / \tilde{\omega}_{p, e}=2.56 \times 10^{-6}\right) \quad$ and $v_{e}=10^{-2} \quad\left(\tilde{v}_{e} / \tilde{\omega}_{p, e}=\right.$ $\left.5.69 \times 10^{-7}\right)$ which gives $T_{0}=66.3\left(\tilde{t} \tilde{\omega}_{p, e}=1.16 \times 10^{6}\right)$. Hence, assuming that only two groups of modes dominate the evolution, this gives a rather good estimate of the period.
Although the characteristics of the beam-driven weak turbulence are of interest, they are not the aim of this study. We rather want to study the spectral signature of such a process on the IS spectrum and to compare it to the observations. The main characteristics for the UHF observations can be summarized as follows (Rietveld et al., 1996):

1. One of the ion-acoustic shoulders of the incoherent spectrum is strongly enhanced by a factor 10 to 100 for 10-s integrated data.

2. The right ion line (upper shoulder) is enhanced more frequently than the left ion line (lower shoulder) at altitudes less than $300 \mathrm{~km}$.

3. Between 300 and $450 \mathrm{~km}$, neither shoulder dominates the statistics.

4. Above $450 \mathrm{~km}$ the lower shoulder is enhanced more frequently.

5. Both shoulders can be simultaneously enhanced above $300 \mathrm{~km}$.

6. Low altitude echoes (i.e. below $200 \mathrm{~km}$ ) are seldom observed.

7. The life time of the coherent echoes is less than 1-s.

Reported observations for the VHF radar lead to similar conclusions (Forme et al., 1995; Forme and Fontaine, 1999).

We have seen that the model presented in this study can enhance either the right, the left or both ion lines simultaneously. The spectral signature of the ISR observations is determined by the beam. The right ion line is easily obtained if the matching condition between the radar backscattered wave vector and the wave vector of the driven Langmuir mode is fulfilled. Using the dimensionless equation corresponding to Eq. (1) we produce a matching condition that can be written as $\mathrm{k}_{V H F} \approx 2 \mathrm{k}_{\max }-1$. What actually selects the wave vector of the driven Langmuir mode is the beam energy $\tilde{E}_{b}$ and the background density $n$. Estimates of the beam energy have been given by Forme (1993) for different background densities.

The left ion line can be enhanced if the ion-acoustic fluctuations corresponding to the second backscatter match $-\mathrm{k}_{V H F}$. This can be achieved for higher growth rates (allowing a second backscatter) and for lower beam energies typically $\tilde{E}_{b} \approx 30 \mathrm{eV}$ for the VHF radar. The first backscatter occurs at $\mathrm{k}_{1}=-\mathrm{k}_{\max }+1$ and the second backscatter at $\mathrm{k}_{2}=\mathrm{k}_{\max }-2$. The ion spectrum corresponding to the second backscatter is centered about $-2 \mathrm{k}_{\max }+3$. Therefore the matching condition for having the left ion line enhanced is $-\mathrm{k}_{V H F}=-2 \mathrm{k}_{\max }+3$. Reducing the beam energy affects the growth rate and therefore we need to increase the beam number density in order reach the second backscatter.

Simultaneous emulation of the two ion lines can be obtained by increasing the energy spread of the beam. It controls the bandwidth, of the excited Langmuir waves $\Delta \mathrm{k}^{l}$ and the ion-acoustic waves $\Delta \mathrm{k}^{s}$. Therefore large energy spread allows us to fulfill simultaneously $\mathrm{k}_{V H F} \approx 2 \mathrm{k}_{\max }-1 \pm \Delta k^{s}$ and $-\mathrm{k}_{V H F}=-2 \mathrm{k}_{\max }+3 \pm \Delta \mathrm{k}^{s}$. Simultaneous excitation of up-going and down-going ion acoustic waves lead to ISR spectra with relative 
amplitude of the two ion lines that vary with time. Hence, the shape of the spectrum appears to be controlled by the duration of the whole process. However, a short time scale leads to very unbalanced spectra. A maximum life time deduced from the observations of enhanced IS spectra is of about $1 \mathrm{~s}$. Forme et al. (1993) calculated the effects of low-frequency turbulence on the thermal structure of the ionosphere. Comparisons between their model and the observations led them to imply a time scale of about $0.1 \mathrm{~s}$ $\left(\tilde{t} \tilde{\omega}_{p, e}=1.78 \times 10^{6}\right)$. Our calculations have been done on for a time scale of about $t=1 \mathrm{~s}\left(\tilde{t} \tilde{\omega}_{p, e}=1.78 \times 10^{7}\right)$. Hence, we believe that the fluctuations of the relative amplitude between the two ion lines shown in Fig. 4 and 5 can affect the shape of the observed spectra.

The beam parameters have their counterpart in the background plasma. Consequently the surrounding plasma can control the shape of the ISR spectra. Changing the beam energy is somehow similar to changing background density. The collision frequency controls the damping of the Langmuir mode and plays a role equivalent to the beam number density. One of the effect of collisions is to balance or inhibit the growth of the Langmuir waves. A large electron collision frequency implies a very large growth rate for parametric decay to occur. In the ionosphere, the electron collision frequency is a function of the altitude, $v_{e}$ decreasing with increasing altitude. One can easily understand that low altitude echoes are rare since the process presented here would need very high growth rates. Also, increasing the electron collisions reduces the bandwidth of the unstable Langmuir waves and therefore it reduces the width in $\mathrm{k}$-space of the growing ion-acoustic waves. It has an effect similar to the energy spread of the beam. Hence, for a given beam energy and energy spread, the probability that this process is detected by a radar decreases as the collision increases. We have seen that small energy spreads (high electron collision frequencies) give preferentially enhancement of the right ion line with respect to the left one. As the energy spread increases (collision decreases), the left and right lines can be balanced. This is consistent with observational points 2, 3 and 5 mentioned. The role of the temperature ratio is to damp the ion-acoustic wave. It is an efficient way of controlling the width of the ion-acoustic spectrum. Large temperature ratios lead to a large ion-acoustic bandwidth and therefore increase the probability of observing the two ion lines being simultaneously enhanced. Also, Forme et al. (1999) have shown that $T_{e}$ can be enhanced by a factor 4 within $10 \mathrm{~s}$ when enhanced ion-acoustic fluctuations are detected. This leads obviously to a time varying spectral signature of the instability. At high altitude, where $\tilde{v}_{e}<50 \mathrm{~s}^{-1}$ our calculation is not valid and another approach, like the numerical calculations of the Zakharov equations, is needed. However, to fit with ISR observations we would expect that the left ion line is enhanced more frequently.

\section{Conclusion}

We have modeled the decay of beam-generated Langmuir waves into ion-acoustic waves using the wave kinetic equations within the framework of the weak turbulence approximation. In this model the driven Langmuir waves are excited by soft electrons drifting in the topside ionosphere. This study was motivated by observations, by means of incoherent scatter radars, of enhanced ion-acoustic fluctuations. We particularly studied the effect of the beam parameters as well as the ionospheric parameters on the incoherent scatter spectrum. We also tried to compare our results to characteristic observations and spectral signatures.

The first formulation of this model by Forme (1993) was not able to explain some of the observed spectral signature like simultaneously propagating ion-acoustic waves in parallel and antiparallel (with respect to the magnetic field) direction. This study shows that the parametric decay of beam generated Langmuir waves can lead to up-going and down-going ion-acoustic waves, propagating separately or simultaneously. The beam energy or the background density selects which ion line (left or right) will be enhanced. A large energy spread of the beam or low electron collision frequencies can explain simultaneous observation of the left and the right ion line. The importance of the electron collision frequency can explain the altitude distribution of the coherent echoes observed by incoherent scatter radars.

Acknowledgement. Topical Editor D. Alcaydé thanks T. Leyser and another referee for their help in evaluating this paper.

\section{References}

Cairns, I. M., A theory for the Langmuir waves in the electron foreshock, J. Geophys. Res., 92, 2329, 1987.

Collis, P. N., I. Häggström, K. Kaila, and M. T. Rietveld, Eiscat radar observations of enhanced incoherent scatter spectra; their relation to red aurora and field-aligned currents, Geophys. Res. Lett., 18, 1031, 1991.

Fontaine, D., and F. Forme, Ionospheric small-scale structures and turbulence inferred from EISCAT observation: a review, J. Geomag. Geoelectr., 47, 869, 1995.

Forme, F. R. E., A new interpretation on the origin of enhanced ion acoustic fluctuations in the upper ionosphere, Geophys. Res. Lett., 20, 2347, 1993.

Forme, F. R. E., and D. Fontaine, Enhanced ion acoustic fluctuations and ion outflows, Ann. Geophysicae., in press, 1999.

Forme, F. R. E., J. E. Wahlund, H. J. Opgenoorth, M. A. L. Persson, and E. V. Mishin, Effects of current driven instabilities on the ion and electron temperatures in the upper ionosphere, J. Atmos. Terr. Phys., 55, 647, 1993.

Forme, F. R. E., D. Fontaine, and J.E. Wahlund, Two different types of enhanced ion acoustic fluctuations observed in the upper ionosphere, J. Geophy. Res., 100, 14 625, 1995.

Foster, J. C., Plasma turbulence and enhanced UHF backscatter from the topside ionosphere, Phys. Space Plasmas, 8, 213, 1988.

Hansen, A., E. Mjølhus, D. F. Dobois and H. A. Rose, Numerical test of the weak turbulence approximation to ionospheric Langmuir turbulence, J. Geophys Res, 97, 12073, 1992.

Ichimaru, S., Basic principles of plasma physics: A Statistical Approach, Benjamin-Cummings, Menlo Park, Calif., 1973.

Kadomtsev, B., Plasma turbulence, Academic Press, London, 1965. 
F. R. E. Forme: Parametric decay of beam-driven Langmuir wave and enhanced ion-acoustic fluctuations

Kindel, J. M., and C. F. Kennel, Topside current instabilities, J. Geophys. Res., 76, 3055, 1971.

Kofman, W., and J.-P. St-Maurice, Non-thermal ionospheric plasma studies using the incoherent scatter technique, J. Atmos. Terr. Phys., 58, 965, 1996.

Muschietti, L., I. Roth, and R. Ergun, Interaction of Langmuir wave packetswith streaming electrons: phase-correlation aspect, Phys. Plasmas, 1, 1008, 1994.

Newman, D. L., M. V. Goldman, and R. E. Ergun, Langmuir turbulence in the auroral ionosphere 2. Nonlinear theory and simulations, J. Geophys. Res., 99, 6377, 1994.

Nicholson, D. R., Introduction to plasma theory, Krieger, Malabar, Florida, USA, 1983.

Payne, G. L., D. R. Nicholson, and Mei-Mei Shen, Numerical test of weak turbulence theory, Phys. Fluids B, 9, 1797, 1989.

Rietveld, M. T., P. N. Collis, and J.-P. St.-Maurice, Naturally enhanced ion acoustic waves in the auroral ionosphere observed with EISCAT 933 MHz radar, J. Geophys. Res., 96, 19 291, 1991.

Rietveld, M. T., P. N. Collis, A. P. van Eyken, and U. P. Lvhaug, Coherent echoes during EISCAT UHF common programmes, J. Atmos. Terr. Phys., 58, 161, 1996.
Robinson, P. A., D. L. Newman, and A. M. Rubenchik, Effects of long-wavelength dissipation on beam-driven Langmuir turbulence, Phys. Fluids B, 8, 2509, 1992.

Rönmark, K., WHAMP-waves in homogeneous, anisotropic multicomponent plasmas, Rep. 179, Kiruna Geophys. Inst., Kiruna, Sweden, 1982.

St.-Maurice J. P., In situ genertion of intense parallel electric fields in the lower ionosphere, J. Geophys. Res., 100, 4533, 1995.

Sagdeev, R. S., and A. A. Galeev, Nonlinear plasma theory, Ed. T. M. O'Neil and D. L. Book, W. A. Benjamin, New York, 1969.

Stasiewicz, K., G. Holmgren, and L. Zanetti, Density depletions and current singularities observed by Freja, J. Geophys. Res., 103, 4251, 1998.

Tsytovich, V. N., Nonlinear effects in plasma, Ed. S. M. Hamberger, Plenum Press, New York-London, 1970.

Zakharov, V. E., Collapse of Lanngmuir waves, Sov. Phys. JETP, (Engl. Transl.), 35, 908, 1972.

Zakharov, V. E., A. N. Pushkarev, A. M. Rubenchik, R. Z. Sagdeev, and V. F. Shvets, Kinetic of three-dimensional Langmuir collapse, Sov. Phys. JETP, (Engl. Transl.), 69, 334, 1989. 